\title{
Editorial
}

\section{Meeting Report: The 7th Leonard Berg Symposium, Part 1}

\author{
Gabrielle Strobel \\ Alzheimer Research Forum
}

\section{SCIENTISTS, FAMILIES TARGET PRECLINICAL DETECTION, TRIALS}

On 1-2 October 2009, 214 researchers from the US, Europe, and Australia, as well as representatives of families with aggressive genetic forms of Alzheimer's disease (AD), met at Washington University, St. Louis, Missouri, to devote two intense days of talks and discussion to their goal of presymptomatic detection of this disease. By a growing consensus, defining the "silent" phase of AD has become the field's central challenge if it is to modernize drug testing and lay the groundwork for AD prevention trials. Presymptomatic carriers of deterministic AD mutations arguably harbor a unique potential to solve this challenge for all of AD. The St. Louis conference pulled together the latest research advances in this area. More than that, it also served as a chance for the far-flung participants in the Dominantly Inherited Alzheimer Network (DIAN) to push their ambitious project forward. In short, initial numbers on enrollment, passionate family input on counseling, community building and data privacy, and budding plans to offer a clinical trial to participating families were the main upshot of these conversations.

In St. Louis, DIAN leaders invited their external advisory committee to review and critique the study's efforts so far. These scientists will send a detailed assessment directly to the study's funder, the National Institute on Aging (NIA), but Thomas Bird of the University of Washington, Seattle, told the Alzheimer Research Forum (ARF) this: "I think the DIAN is a good idea and will provide valuable information. As I see it, the major goal is to identify the earliest signs, symptoms, and biomarker changes in persons known to be at essentially 100 percent risk for developing AD. This data will be important for understanding the biology of the disease and organizing appropriate therapeutic trials. The challenges are several: 1) recruiting and retaining an informative number of subjects, 2) choosing the "right" biomarkers, and 3) demonstrating that the information is relevant to the common forms of AD occurring in the general population."

Presymptomatic detection in dominantly inherited $\mathrm{AD}$ became this year's topic of the 7th Leonard Berg Symposium, a biennial conference at WashU that began in 1997. The symposium honors the late Leonard Berg, the founder of the WashU Alzheimer Disease Research Center. A clarinetist and saxophone player, Berg finished medical school in his native town of St. Louis at age 22 and built a life of pioneering clinical and advocacy work from there. Berg developed the widely used dementia staging instrument, Clinical Dementia Rating (CDR), which uses informant interviews and intra-individual change as important components. As early as 1979, Berg led the launch of large, decades-long natural history studies in AD and normal aging that are ongoing at WashU. Now led by John Morris of WashU, these studies have spun off longitudinal biomarker studies in the second generation of research participants and in families with autosomaldominant AD. Berg succumbed to strokes caused by amyloid angiopathy in 2007 [1].

The symposium combined a scientific update on catching presymptomatic AD in its genetic and spo- 
radic forms with discussion on whether the former models the latter, and a nuts-and-bolts meeting among the leaders of DIAN. This initiative is building an intercontinental, 10-center registry of mutation carriers and their non-carrying relatives, who are adult children of a parent with a known causative mutation in the $\mathrm{A} \beta \mathrm{PP}$ or presenilin genes. It aims to pull these rare and dispersed families together into a longitudinal study that establishes the biological and cognitive course of their preclinical period with standardized data. Importantly, it tries to do so with a degree of statistical power that can support future prevention and treatment trials relevant to the families themselves and also to the much larger population of late-onset AD. ARF has described the rationale and the structure of this complex undertaking before; hence, this set of stories will focus on what's new since then.

Chief among the news, DIAN is up and running. Twenty-one participants to date have completed the first round of assessments. As often happens with multicenter studies, enrollment was slow off the blocks, even as prospective participants were impatiently waiting as centers worked to obtain the necessary IRB approvals and local certifications, investigators and some family members said. Like with the Alzheimer's Disease Neuroimaging Initiative (ADNI), for example, each DIAN site needs to be certified on Pittsburgh compound B (PIB) synthesis on best practices, an imaging phantom has to be sent around to ensure standardization of the magnetic resonance imaging (MRI) measurements, etc. This has taken the better part of this year at many sites, but five sites (WashU; Brigham And Women's Hospital; Brown; University of New South Wales in Sydney, Australia; and UCLA) are enrolling already and all others will begin within the month, said Morris, the principal investigator for DIAN. The goal now is to have 100 people enrolled by next July, when the DIAN leaders will gather for their forthcoming face-to-face meeting at the next International Conference on Alzheimer's Disease (ICAD) conference in Honolulu, Hawaii, and 150 by end of 2010. "This number would put us on track with the original aims of the grant application," Morris added.

The next ICAD conference also is a goalpost for perhaps the biggest new development at DIAN. At WashU last week, its leaders approved formation of a Clinical Trials Committee, which in plain English means they are putting their thinking caps on for real. Offering drug trials, either for prevention and/or treatment of early symptoms, has always been very much in the investigators' minds, Morris said. After all, that's what the families ultimately want and where the potential lies for late-onset $\mathrm{AD}$, as well. But the investigators first needed to focus on getting DIAN going, and felt it unethical to mention future trials as part of what is primarily an observational study when it was not clear yet whether DIAN would happen or whether companies would show sufficient interest. In practice, DIAN does not have enough participants to begin a trial within the next 12 months. However, preparing for such trials requires a lot of work because challenges about design, choice of drug, and genetic privacy need to be overcome [2]; hence, the time to start planning is now.

Randall Bateman of WashU heads this group. "People at the steering committee meeting were very enthusiastic about starting to explore how clinical trials could be done soon," said Bateman. At the Leonard Berg symposium, Bateman, Nick Fox of University College, London, UK, John Ringman of the University of California, Los Angeles, Stephen Salloway of Brown University in Providence, Rhode Island, and Reisa Sperling of Boston's Brigham and Women's Hospital discussed how best to engage drug company researchers to ensure both scientific rigor in design and execution, and independence in selecting the best drug candidate. "The hope is to have a full group, a charter, and a process in place by next July, so concrete design can go from there," Bateman said.

During the Leonard Berg Symposium, the spouse of an eFAD patient was asked whether her adult children would want to participate in a treatment or even a prevention trial. Unsurprisingly, perhaps, the answer was: "They would try anything. Their outlook is so bleak. They worry less about side effects from an experimental treatment than about AD."

A separate goal where DIAN is moving more slowly is expanding the network to include more sites. From the get-go, researchers in Spain, Sweden, Japan, and Italy, who have for years cared for and studied families with autosomal-dominant $\mathrm{AD}$, expressed interest in joining DIAN. "We are open to expanding DIAN but are not ready yet. We first have to focus on getting all 10 sites going full steam on the existing protocol," Morris said. Taking on more sites and more languages will add complexity and cost. One delicate question is whether sites would be added to the 10 present ones or whether a performance review might swap out underperforming sites for new ones. In St. Louis, the DIAN leaders decided to postpone the issue for one year. That said, some sites have a lot to offer the network if at least a Spanish language version of DIAN could be prepared, Morris noted. Two existing sites, Columbia 
University and UCLA, are working with Puerto Rican and Mexican families, respectively, and in the scientific session of the symposium, Raquel Sanchez-Valle of the Hospital Clinic Villarroel in Barcelona, Spain, reported that her team is already working with several dozen at-risk relatives from nine eFAD families.

DIAN itself is in large part about the families in the network. The study will be more successful if it actively integrates them into a shared sense of commitment. To this end, DIAN reserves two seats on its steering committee to family representatives. In St. Louis, an at-risk daughter and a caregiver and mother of six made their mark in two areas. First, they insisted that data sharing has to be devised with the utmost concern for their privacy. "They are steadfast on this issue," Morris said. The ADNI, on which DIAN is modeled, prides itself on making its data available almost instantly to all qualified researchers worldwide as soon as it enters the system. DIAN cannot do that. The investigators have to ensure that the data cannot accidentally identify families or unmask individuals within a given family. For example, study participants who chose not to know their carrier status must be unable to recognize their scan and realize they have amyloid in their brains. Therefore, the goal is to refine de-identification procedures and release that data within at most a year of its deposition in the DIAN database. "That said, we do intend to release all data, not just bits and pieces. But the guiding concerns have to be confidentiality, confidentiality, and confidentiality," Morris quipped.

Secondly, the family representatives made a case for psychological and emotional support. This aggressive form of $\mathrm{AD}$ can divide extended families as much as it sometimes unites others. Stigma, fear, and the sheer burden of caregiving drive them into isolation. To address these needs, DIAN formed a participant liaison committee. It will work on communicating with participants and supporting them with online services. One idea is to create a forum where they can network and talk about their experiences with AD and DIAN, without the DIAN investigators being privy. "We know we ask a great deal of the study participants. We want to do things not only appropriately but also give them the necessary psychological and social support," Morris said. Martin Rossor of University College London, UK, who has taken care of families with dominantly inherited AD for 20 years and is now seeing adult children of his original patients, will head this participant liaison group.

When asked whether support services were important, a different, unaffected spouse and mother of three adult children at risk for eFAD wrote to ARF: "Psychologic counseling, above all genetic counseling, are extremely important. The decision to find out one's fate can be daunting, especially in the case of FAD where there is no cure. I have seen that spouses, siblings, or parents may try to influence participants to be tested or not, but ultimately it should be the decision of the possible carriers, and counseling helps them make that call. Social/emotional support from others going through the same nightmare is as important as counseling. Most families don't know other families facing similar struggles because the genetic AD mutations are so rare. Most DIAN participants have been caregivers to their own parent or other relatives and know all too well the devastation Alzheimer's causes to the afflicted individual and the entire family unit. I would hope for call-in support groups, but if that is not possible, then an online forum would be the next best thing."

In other DIAN news, the scientists decided to add a new sequence to the MRI component, i.e., diffusion tensor imaging. This was not part of the initial protocol but may help the scientists understand if and when the brain's white matter changes in the run-up to AD. This will not add imaging time or the number of sessions for participants.

Finally, DIAN has a resource allocation review panel headed by David Holtzman, through which scientists interested in studying cerebrospinal fluid (CSF) or plasma samples can request access. Holtzman, like all DIAN investigators, sites, and partnering groups, can be contacted through DIAN.

\section{THE FAMILY VIEW - WHAT DO STUDY VOLUNTEERS WANT FROM DIAN?}

Scientists experiment in laboratories, doctors diagnose and adjust medications, company folks make drugs and run trials, and caregivers deal with the disease at home. People work, often extremely hard, in their respective corner of the Alzheimer's world, yet they rarely come together under one roof to tell the others what it's really like. In particular, when do researchers get to hear from study participants how deeply their families have struggled for decades and what kinds of services would make the repeated poking, measuring, and scanning of a six-year research study well worth their while?

Exactly such a conversation took place at the 7th Leonard Berg Symposium held 1-2 October 2009 at Washington University, St. Louis, Missouri. Unsur- 
prisingly, perhaps, this hour stirred much more emotion than do the usual proceedings in scientific auditoriums. As two family representatives briefed the audience, both their tears and their dignity prompted spontaneous applause and standing ovations from the 214 people in attendance, most of them scientists.

The two women have seats on the steering committee of DIAN, and in this role help shape this study as it unfolds. In their talks (see below), they urged DIAN researchers to find ways to engage the participating families in broader ways. They made clear that having dominantly inherited AD reappear in generation after generation casts a wrenching pall on all family members, not just the affected relatives. Consequently, DIAN would do well to provide psychological and emotional counseling and support for people at risk for dominantly inherited AD. This could be done in person, through call-in support groups, or through protected social networking forums that build a virtual community for study participants spread across the globe. If this kind of support accompanied the study itself, one caregiver said, families would view DIAN participation as less stressful, would become invested in the effort, and more readily return for assessments. Families also need practical advice on issues such as obtaining long-term care insurance policies or persuading the Social Security agency that their loved ones qualify for disability even if they are only 45 and can still walk and talk. For their part, DIAN scientists said they would explore ways for DIAN participants to connect and speak openly about their experience without having to go through the DIAN investigators. Below are excerpts of their presentations.

\section{FAMILY REPRESENTATIVE 1 (NAME WITHHELD)}

I am showing you here a picture of my grandmother and my mother. And here's my special auntie, mom's oldest sister. All three were teachers. I am a teacher. All have had AD. I am at risk.

I am 40, a wife, a mom of three young boys. I came to the US on a scholarship to study special education and currently am a full-time teacher. I worked parttime in my state's chapter of the Alzheimer's Association, doing outreach to Latino families. Then that was becoming too much - to deal with AD all day at work and go home and deal with it personally. I needed to go back to a more normal life.
I am mother, daughter, sister, niece, granddaughter, cousin - and the disease marks all these relationships. I work a lot on my family genealogy and it, too, is threaded through with this disease.

I started to learn about $\mathrm{AD}$ from my grandmother. I did not know her well. She was in her early sixties and already very sick when I was a child. The onset in my family is in the fifties. We played school and I thought it was a game, but mom told me that granny really believed we were her students.

I had an inkling about my mom very early on in her course. That was terrible, because I'd seen the emotional toll the disease took on my mom, who cared for grandma while also blaming her. I helped take care of grandma. One of my secrets for many years was that I was relieved when she died. I was 14 and felt so very guilty. To this day this memory makes me cry. But she was granted the gift of death. In my family the disease runs its course for some 20 years after it has fully manifested. It initially moves slowly, and that is good for us because we get to have our loved ones with us longer. But the final dreadful stages drag on for a long time.

Alzheimer disease was stigmatized. We took care of grandma without talking about it. I found that people were hiding their sick family members. Not intentionally, but you just did not talk about it. Even in my own extended family, people have gone through it alone all the way to death. They did not know, and did not want to know, that other relatives were going through the same thing, living just a few miles away.

In my family, people believe it is a curse visited on the family. That's common in my culture. But even independent of culture, fear, paranoia, and confusion, are all very common in families in our situation.

We need support to deal with those feelings, particularly when we decide to participate in a research study and face our risk and fears head-on. I would welcome counseling or a support group for people like me. There is not much out there for us.

By participating in DIAN, we are trailblazing the way into a new era of medicine. But we also need research on the psychological impact on families of autosomal-dominant Alzheimer disease. I don't know if I have the gene. No one in my family has found out. I have not because I would not do anything differently if I knew. My sister wants to know yesterday. I will want to know at some point in the future.

What do the families participating in DIAN need? Counseling. Psychological and emotional support. Please offer it throughout the length of the study. To 
the researchers, I say: I am sure that if you build a good relationship with us, we will want to come back. Taking care of us in this way can prevent catastrophic outcomes.

Around our coffee table, we are afraid of loss of insurance. We need guidance on how to navigate that issue. We are afraid of breach of privacy - please handle our data with the greatest care.

I spend a lot of time wondering how my participation in such a study will affect my children in the future? Please take time thinking this through with us, and explaining where you can.

From our perspective, anything and everything that makes the process more comfortable or less stressful will help the study be a success.

\section{DENISE HEINRICHS}

My husband Vince and I started our search for understanding AD in the 1960s. His mom had had it. She was diagnosed with 'hardening of the arteries,' the same diagnosis given to her own mom and three brothers before. Vince knew that was not it. They had died at an early age in state mental institutions or nursing homes. The relatives in his mom's generation refused to help us. They would not talk about a mental illness. We got more help from the next generation, when several cousins died in their forties and fifties. They allowed autopsies and we found out the genetic cause.

Then in the early 1980s, I noticed Vince's problems. He was 39 and a college teacher. Four years later he could no longer teach. We have six children. He enrolled in an NIH study right away and got evaluated every six months. He did every test they had available at that time. At his first visit in 1983 his IQ was 136; by his last visit in 1987 it was below 50 .

He learned all he could about AD. When he could no longer drive, he rode his bike around town. It was hard for him to give up his car, but he had gotten into an accident. He read and we played Scrabble as much as we could to preserve what was still there.

It was not easy. Our children were very aware. They were teenagers, with all the typical teenage issues, and the youngest was in kindergarten when Vince got sick. He first read to her, then she read to him when he no longer could.

He got very angry. As with every large family, there was a lot of noise and commotion in our house, and he could not cope with that. It got hard to care for him. Stress and noise upset him. He developed seizures. In
1987, we had to place him in a nursing home. He no longer knew us and had become violent with the kids and me. That was exactly what he'd worried about early on.

By this time, his brother already was in a nursing home with a feeding tube and was being treated for pneumonia three, four times a year. This went on many years. Vince had seen this in the years before and decided against such treatment. I made sure this would not happen to him. I obtained power of attorney and made clear that we wished no treatment other than comfort. Thankfully, he got pneumonia at some point and passed soon after surrounded by loving care and his family.

My children are brave. They will be part of the DIAN study. I have grandchildren. My brother-inlaw's children, of course, are facing this issue, too, as are many more in Vince's extended family.

Alzheimer disease has consumed half of my lifetime. The toll on families is devastating, causing many additional illnesses. One problem is getting the affected on disability. This can take over a year. The wait causes immense stress and cost, and worry about losing the home or meeting day-to-day expenses. That can be overwhelming.

I feel helplessness, sadness, fear, anger, and gratitude. I am helpless and sad for my children. I am angry because for years I have been told the cure is 10 years away, but that has not been true. I am angry as more of us get diagnosed. I am grateful for my family. I have found that family support is crucial. The parts of my extended family that support each other actively and openly are doing so much better. I am also grateful to be involved in research that is going on. We will continue to give until the answer is found. We will go anywhere and do anything until a treatment is found to stop this horrible disease. We understand it takes research participation to get there.

And here is a third voice, from a caregiver and mother of three who brought her affected husband to the symposium. When asked what DIAN could do to ensure that her children would want to enroll and keep returning for visits and invasive procedures, she said: "The single most important thing DIAN has to do to ensure return visits is to offer continued hope for the future of a world without Alzheimer's, or at least the ability to delay the onset of the disease. The researchers and participants need to have open lines of communication and form a partnership working together for the common goal. Part of offering hope is allowing participants the first opportunity for new treatments that have 
been deemed safe. As an unaffected parent, I have encouraged my children to participate because I feel it is their responsibility to themselves, their families, their communities, and the world."

Vice versa, the Leonard Berg Symposium also taught the attending families about the complexity of the science underlying dominantly inherited $\mathrm{AD}$, as well a drug company perspective on the promise and challenges of designing treatment and prevention trials for their loved ones.

\section{REFERENCES}

[1] Morris JC, Landau WM (2007) Leonard Berg, MD (19272007). Neurology 69, 1206-1207.

[2] Ringman JM, Grill J, Rodriguez-Agudelo Y, Chavez M, Xiong C (2009) Commentary on "a roadmap for the prevention of dementia II: Leon Thal Symposium 2008." Prevention trials in persons at risk for dominantly inherited Alzheimer's disease: opportunities and challenges. Alzheimers Dement 5, 166-171. 